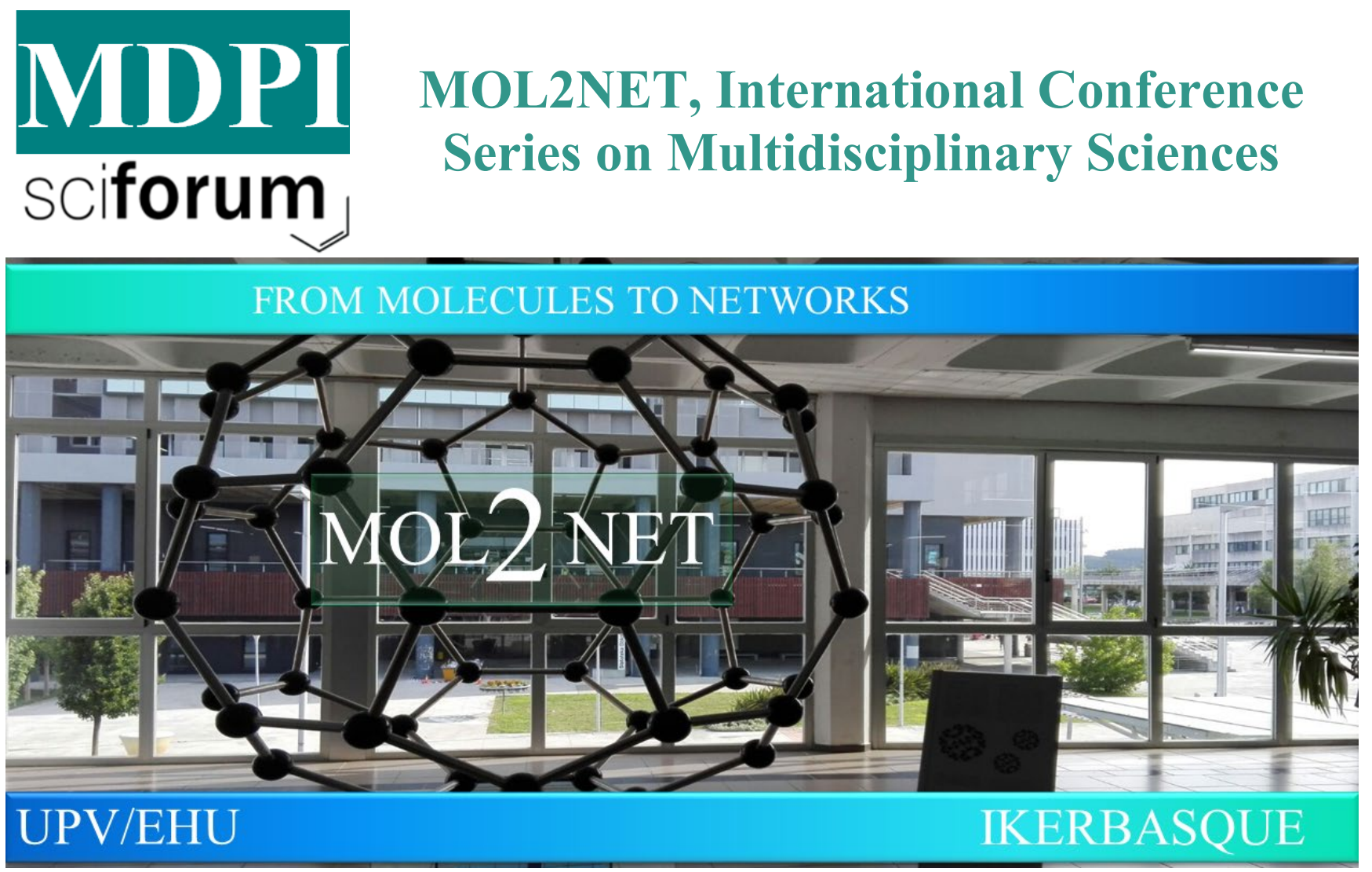

\title{
Changes in the expression pattern of genes encoding selected adipokines in endometrial cancer
}

Robert Kietbasiński ${ }^{a}$, Beniamin Oskar Grabarek ${ }^{a}$, Krzysztof Balawender ${ }^{b}$, Dariusz Boroń $^{a}$

${ }^{a}$ Department of Histology, Cytophysiology and Embryology, Faculty of Medicine in Zabrze, University of Technology in Katowice, Poland

${ }^{b}$ Morphological Sciences Department of Human Anatomy, Institute of Medical Sciences, Medical College of Rzeszow University, 35-959 Rzeszów, Poland

\section{Graphical Abstract}

\begin{abstract}
.
Leptin (LEP) is primarily secreted by differentiated adipocytes, influencing neoplastic angiogenesis through the activation of the JAK/STAT pathway, which results in the increased proliferation of vascular endothelial cells as well as an increased expression of the vascular endothelial growth factor (VEGF),
\end{abstract}




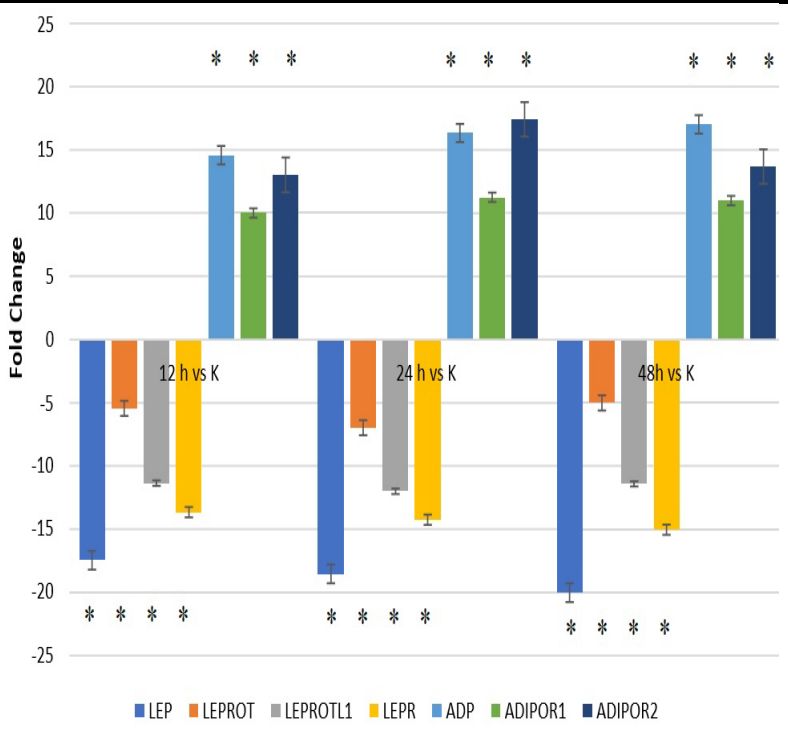

Figure 1. Expression of adiponectin, leptin and their receptors depending on the exposure time of the Ishikawa cell line to cisplatin. fibroblast growth factor (FGF) and antiapoptotic proteins, namely Bcl-2. In women whose BMI is higher than 25, the risk of endometrial cancer is doubled and in those whose BMI is above 30, this risk is tripled. A higher concentration of LEP in the serum of women with endometrial cancer compared to healthy volunteers was also confirmed.

In turn, adiponectin (ADP) demonstrates an insulin-sensitizing effect, increasing the expression of nitric oxide synthase, furthermore, it also has anti-inflammatory activity through inhibiting the expression of tumor necrosis factor-alpha (TNF- $\alpha)$ and interleukin 6 (IL-6). A 6-times higher risk of cancer development is noted in obese people, in whom the concentration of ADP was lower than in the group of healthy volunteers. It is highlighted that it is this adipokine that is most strongly associated with the risk of endometrial cancer.

The overriding aim of this study was the assessment of changes in the expression pattern of genes coding chosen adipokines in an Ishikawa line endometrial cancer cell culture exposed to the effects of cisplatin, compared to a control culture.

Ishikawa line endometrial cancer cells were exposed to the effects of cisplatin at concentrations of $2.5 \mu \mathrm{M}, 5 \mu \mathrm{M}$, and $10 \mu \mathrm{M}$ for 12, 24, and 48 hours, and afterward compared to a control culture (C), which consisted of cells untreated using cisplatin. For each exposition period and cisplatin concentration, 3 technical repetitions were conducted.

From the results of the microarray experiment, it can be observed that under the influence of cisplatin, there is a decrease in the transcriptional activity of leptin and its' three receptors. A lower expression of the discussed genes was noted, the higher the concentration of the drug added to the culture and the higher the exposition time of the cells to the drug was $(p<0.05)$. 
In turn, for adiponectin and its' receptors, an opposite expression profile to leptin and its' receptors is noted, depending on the cisplatin concentration and treatment time of the endometrial cancer cells using it. The higher the drug concentration and cell exposition time, the higher the overexpression of adiponectin and its' receptors.

Changes in the expression profiles of leptin, adiponectin, can be explored in the context of utilizing them as supplementary diagnostic markers of endometrial cancer, monitoring the effectiveness of cisplatin therapy, and in the creation of new therapeutic strategies aimed toward the JAK/STAT signaling pathway.

\section{Introduction (optional)}

Leptin (LEP) is primarily secreted by differentiated adipocytes, influencing neoplastic angiogenesis through the activation of the JAK/STAT pathway, which results in the increased proliferation of vascular endothelial cells as well as an increased expression of the vascular endothelial growth factor (VEGF), fibroblast growth factor (FGF) and anti-apoptotic proteins, namely Bcl-2. In women whose BMI is higher than 25, the risk of endometrial cancer is doubled and in those whose BMI is above 30 , this risk is tripled. A higher concentration of LEP in the serum of women with endometrial cancer compared to healthy volunteers was also confirmed.

In turn, adiponectin (ADP) demonstrates an insulin-sensitizing effect, increasing the expression of nitric oxide synthase, furthermore, it also has anti-inflammatory activity through inhibiting the expression of tumor necrosis factor-alpha (TNF- $\alpha$ ) and interleukin 6 (IL-6). A 6-times higher risk of cancer development is noted in obese people, in whom the concentration of ADP was lower than in the group of healthy volunteers. It is highlighted that it is this adipokine that is most strongly associated with the risk of endometrial cancer.

The overriding aim of this study was the assessment of changes in the expression pattern of genes coding chosen adipokines in an Ishikawa line endometrial cancer cell culture exposed to the effects of cisplatin, compared to a control culture.

\section{Materials and Methods (optional)}

Ishikawa line endometrial cancer cells were exposed to the effects of cisplatin at concentrations of 2.5 $\mu \mathrm{M}, 5 \mu \mathrm{M}$, and $10 \mu \mathrm{M}$ for 12,24 , and 48 hours, and afterward compared to a control culture (C), which consisted of cells untreated using cisplatin. For each exposition period and cisplatin concentration, 3 technical repetitions were conducted.

\section{Results and Discussion (optional)}

From the results of the microarray experiment, it can be observed that under the influence of cisplatin, there is a decrease in the transcriptional activity of leptin and its' three receptors. A lower expression 
of the discussed. In turn, for adiponectin and its' receptors, an opposite expression profile to leptin and its' receptors is noted, depending on the cisplatin concentration and treatment time of the endometrial cancer cells using it. The higher the drug concentration and cell exposition time, the higher the overexpression of adiponectin and its' receptors.

\section{Conclusions (optional)}

Changes in the expression profiles of leptin, adiponectin, can be explored in the context of utilizing them as supplementary diagnostic markers of endometrial cancer, monitoring the effectiveness of cisplatin therapy, and in the creation of new therapeutic strategies aimed toward the JAK/STAT signaling pathway.

\section{References (mandatory)}

1. Fève B, Bastard C, Fellahi S, Bastard JP, Capeau J. New adipokines. In Annales d'endocrinologie. Elsevier Masson. 2016; 77(1): 49-56.

2. Friedman J. The long road to leptin. J Clin Invest 2016; 126(12): 4727-4734.

3. Izquierdo AG, Crujeiras AB, Casanueva FF, Carreira MC. Leptin, obesity, and leptin resistance: where are we 25 years later?. Nutrients 2019; 11(11): 2704.

4. Friberg E, Mantzoros CS, Wolk A. Diabetes and risk of endometrial cancer: a population-based prospective cohort study. Cancer Epidemiol Biomarkers Prev. 2007; 16(2): 276-280.

5. Rzepka-Górska I, Bedner R, Cymbaluk-Płoska A, Chudecka-Głaz A. Serum adiponectin in relation to endometrial cancer and endometrial hyperplasia with atypia in obese women. Eur J Gynaecol Oncol. 2008; 29(6): 594-597.

6. Makovec T. Cisplatin and beyond: molecular mechanisms of action and drug resistance development in cancer chemotherapy. Radiol Oncol. 2019; 53(2): 148-158. 